\title{
Grief and Bereavement: Theoretical Perspectives
}

\author{
Kathrin Boerner $^{\mathrm{a} *}$, Margaret Stroebe ${ }^{\mathrm{b}}$, Henk Schut ${ }^{\mathrm{b}}$ and Camille B. Wortman ${ }^{\mathrm{c}}$ \\ ${ }^{a}$ Department of Gerontology, University of Massachusetts Boston, Boston, MA, USA \\ ${ }^{b}$ Department of Clinical and Health Psychology, Utrecht University, Utrecht, The Netherlands \\ ${ }^{\mathrm{c}}$ Department of Psychology, SUNY Stony Brook, Stony Brook, NY, USA
}

\section{Synonyms}

Deep sorrow (caused by someone’s death); Mourning (expression of grief); Personal loss

\section{Definition}

The term bereavement denotes the objective situation of a person who has experienced the death of someone significant. Grief then refers to the emotional experience of the psychological, behavioral, social, and physical reactions the bereaved person might experience as a result of this death.

\section{Introduction}

The loss of a loved one is a ubiquitous human experience, which is often regarded as a serious threat to health and well-being. This topic is relevant to the study of geropsychology for two reasons. First, there is consensus among researchers and practitioners alike that coming to terms with personal loss is a critical part of successful adult development (Baltes and Carstensen 1996). Second, losses tend to accumulate in late life. This can place survivors in a state of chronic stress and undermine their capacity to deal with any particular loss.

The death of a loved one provides an excellent arena to study basic processes of stress and adaptation to change. Unlike many stressful life experiences, the death of a person is irrevocable and cannot be altered by the coping efforts of survivors. Indeed, the major coping task faced by those who have experienced the death of a loved person is to reconcile themselves to a situation that cannot be changed and find a way to carry on with their own lives.

The most common loss of a loved one for older adults is the death of a spouse (Carr et al. 2006). Therefore, the bulk of research on late life losses has focused on this type of loss. However, older adults may also experience other types of losses including the death of a child, grandchild, sibling, or close friend. These can also be major losses and may each have unique implications and challenges for the person who is faced with them. Research insights shedding light on these experiences, however, are much more limited. It is thus important to keep in mind that much of our understanding of and thinking about late life losses is derived from work on spousal loss.

Furthermore, it is important to define the key terms that will be used throughout this entry. The term bereavement is derived from the Latin word rumpere (to break, to carry, or tear away) and refers to the objective situation of a person who has suffered the loss of someone significant. In most cases, bereavement robs survivors of love and companionship, as well as future hopes and plans that they expected to share with the person who died. Grief is derived from Latin gravare (to weigh down) and

\footnotetext{
*Email: kboerner@jewishhome.org
} 
refers to the emotional experience of a number of psychological, behavioral, social, and physical reactions to one's loss. The word mourning is derived from the Latin word memoir (mindful). It refers to actions expressive of grief which are shaped by social and cultural practices and expectations. Pointing to the timeless message of the original meanings of these terms, Jeter (1983) commented that "as the ancients, people today surviving the death of a family member do feel robbed, weighted down, and are mindful of the past, knowing that life will never be the same" (p. 219). But how do individuals cope with such an experience? To address this question, models and approaches that seem most influential to current understanding of bereavement and grief are examined.

\section{Classic Psychoanalytic View}

One of the most influential approaches to loss has been the classic psychoanalytic model of bereavement, which is based on Freud's seminal paper, "Mourning and Melancholia" (1917). According to Freud, the primary task of mourning is the gradual surrender of one's psychological attachment to the deceased. Freud believed that relinquishment of the love object involves a painful internal struggle. The individual experiences intense yearning for the lost loved one, yet is faced with the reality of that person's absence. As thoughts and memories are reviewed, ties to the loved one are gradually withdrawn. This process, which requires considerable time and energy, was referred to by Freud as "the work of mourning." At the conclusion of the mourning period, the bereaved individual is said to have "worked through" the loss and to have freed himself or herself from an intense attachment to the unavailable person. Freud maintained that when the process has been completed, the bereaved person regains sufficient emotional energy to invest in new relationships and pursuits. This view of the grieving process has dominated the bereavement literature over much of the past century and only more recently has been called into question (Bonanno and Kaltman 1999; Stroebe 1992; Wortman and Silver 1989). For example, it has been noted that the concept of grief work is overly broad and lacks clarity because it fails to differentiate between such processes as rumination, confrontative coping, and expression of emotion (Stroebe and Schut 2001).

\section{Attachment Theory}

Another theoretical framework that has been extremely influential is Bowlby's attachment theory (Bowlby 1969, 1973, 1980; see also Fraley and Shaver 1999; Shaver and Tancredy 2001). In this work, Bowlby integrated ideas from psychodynamic thought, from the developmental literature on young children's reactions to separation, and from work on the mourning behavior of primates. Bowlby maintained that during the course of normal development, individuals form instinctive affectional bonds or attachments, initially between child and parent and later between adults. He believed that the nature of the relationship between a child and his or her mother or caregiver has a major impact on subsequent relationships. He suggested that when affectional bonds are threatened, powerful attachment behaviors are activated, such as crying and angry protest. Unlike Freud, Bowlby believed that the biological function of these behaviors is not withdrawal from the loved one but rather reunion. However, in the case of a permanent loss, the biological function of regaining proximity with attachment figures becomes dysfunctional. Consequently, the bereaved person struggles between the opposing forces of activated attachment behavior and the reality of the loved one's absence.

Bowlby maintained that in order to deal with these opposing forces, the mourner goes through four stages of grieving: initial numbness, disbelief, or shock; yearning or searching for the deceased, accompanied by anger and protest; despair and disorganization as the bereaved gives up the search, accompanied 
by feelings of depression and hopelessness; and reorganization or recovery as the loss is accepted and there is a gradual return to former interests. By emphasizing the survival value of attachment behavior, Bowlby was the first to give a plausible explanation for responses such as searching or anger in grief. Bowlby was also the first to maintain that there is a relationship between a person's attachment history and how he or she will react to the loss of a loved one. For example, children who endured frequent separations from their parents may form anxious and highly dependent attachments as adults and may react with intense and prolonged grief when a spouse or partner dies (see Shaver and Tancredy (2001), or Stroebe et al. (2005), for a more detailed discussion). Because it provides a framework for understanding individual differences in response to loss, Bowlby's attachment model has continued to be influential in the study of grief and loss (Shear et al. 2007). Mikulincer and Shaver (2013) have conducted sophisticated empirical research, confirming the importance of attachment security in the prediction of adjustment to bereavement and providing fine-grained understanding of many associated phenomena.

\section{Getting Past Stage of Grief}

While several theorists have proposed that people go through stages or phases in coming to terms with loss (see, e.g., Bowlby 1980; Horowitz 1986), perhaps the most well known of these models is the one proposed by Kübler-Ross in her highly influential book On Death and Dying (Kübler-Ross 1969). This model, which was developed to explain how dying persons react to their own impending death, posits that people go through denial, anger, bargaining, depression, and ultimately acceptance. It is Kübler-Ross's model that popularized stage models of bereavement. For many years, Kübler-Ross's model has been taught in medical, nursing, and social work schools. It has also appeared in articles in newspapers and magazines written for bereaved persons and their family members. As a result, stage models have strongly influenced the common understanding of grief in our society.

As research began to accumulate, it became clear that there is little support for the view that there are systematic stages. Although there are studies that purport to support stage models (Maciejewski et al. 2007), the weight of the evidence suggests that reactions to loss vary considerably from person to person and that few people pass through the stages in the expected fashion (see Archer 1999; Attig 1996, for a review). Several major weaknesses of stage models have been identified (Neimeyer 1998). First, they cannot account for the variability in response that follows a major loss. Second, they place grievers in a passive role when in fact grieving requires the active involvement of the survivor. Third, such models fail to consider the social or cultural factors that influence the process. Fourth, stage models focus too much attention on emotional responses to the loss and not enough on cognitions and behaviors. Finally, stage models tend to pathologize the reactions of the majority of people who do not pass through the stages. As a result of these and other critiques and a lack of empirical support, most researchers have come to the conclusion that the idea of a sequence of stages is not particularly useful (Stroebe et al. 2001).

\section{Trauma Theory and Meaning Making}

An influential stream of thought in the field of bereavement has come from trauma theory. Even though one could argue that most late life losses may not involve experiences of a traumatic nature, for example, because the occurrence of the death may be considered timely in the context of the life course, there could be traumatic aspects to a loss experience in late life. For example, witnessing a prolonged period of serious illness and intense suffering of a loved one can have elements of trauma, even if the illness and death occur at an expected life stage. Similarly, the death of an older person can be experienced as very sudden, if this 
person had been in good health and highly engaged in life or even if the person died after years of chronic illness and decline. Therefore, it appears that conceptual thinking coming from trauma theory should be considered even in the context of late life loss.

The model of stress response syndromes by Horowitz and colleagues (1986) purports that traumatic experiences disrupt a person's life via blockage of cognitive and emotional processing. Similar to the notion of grief work as a necessary step toward recovery, the assumption here is that processing the trauma is essential if the person is going to be able to move on and that stressful life events play an important role in the etiology of various somatic and psychiatric disorders due to failure of such processing. A further line of research derived from the related field of trauma was that of Janoff-Bulman (1992), particularly through the identification of shattered beliefs which need to be rebuilt. This has been expanded to the study of "meaning making" particularly by Neimeyer and collaborators $(2001,2006)$.

The basic idea in the perspective is that major losses challenge a person's sense of identity and narrative coherence. Narrative disorganization can range from the relatively limited and transient to more sweeping and chronic, depending on the nature of the relationship and the circumstances surrounding the death. According to Neimeyer, a major task of grief involves reorganizing one's life story to restore coherence and maintain continuity between the past and the future. However, difficulties in establishing the role of meaning making in adjustment remain (e.g., studies have not always succeeded in separating the process from the outcome, beliefs from adjustment, or establishing the direction of causality among these factors). Others have distinguished two components of meaning making. Davis, Nolen-Hoeksema, and Larson (1998) identified two distinct processes, making sense of the loss and finding benefit, which entail distinguishable psychological concerns for the bereaved person, with, for example, the former diminishing in importance in time, while the latter grows stronger as time goes on.

\section{Stress and Coping Approach}

Over the past two decades, a theoretical orientation referred to as the stress and coping approach, or the cognitive coping approach (Lazarus and Folkman 1984), has become highly influential in the field of bereavement. Stress and coping theorists maintain that life changes such as the death of a loved one become distressing if a person appraises the situation as taxing or exceeding his or her resources. An important feature of this model is that it highlights the role of cognitive appraisal in understanding how people react to loss. A person's appraisal, or subjective assessment of what has been lost, is hypothesized to influence his or her emotional reaction to the stressor and the coping strategies that are employed. To explain why a given loss has more impact on one person than another, stress and coping researchers have focused on the identification of potential risk factors, such as a history of mental health problems, as well as protective factors, such as optimism or social support (for a review, see Hansson and Stroebe 2007; Pearlman et al. 2014; Stroebe et al. 2006, 2007). The appraisal of the loss, as well as the magnitude of physical and mental health consequences that result from the loss, are thought to depend on these factors.

\section{Caregiving and Bereavement}

As most deaths in late life are preceded by chronic illnesses, family members, in particular spouses, are often involved in prolonged periods of caregiving in the years, months, or weeks before their loved one's death. Therefore, conceptual thought that considers the specific case of bereavement after caregiving is particularly relevant to the topic of late life loss. Three major lines of thought derived from stress theory 
have emerged in the literature (for a review, see Boerner and Schulz 2009; Schulz et al. 2008) regarding bereavement in the context of caregiving. The cumulative stress perspective, or "wear and tear" hypothesis, argues that the combined effects of the stress of caregiving and the death deplete people's coping resources and result in greater adjustment difficulties following the loss. The stress reduction perspective makes the opposite prediction, arguing that the death brings relief because it puts an end to caregiving stressors and the suffering of the person who is dying and so results in more positive bereavement outcomes than found among non-caregivers. Finally, it has been suggested that caregivers more or less expect to be bereaved, which in turn allows at least some degree of anticipatory processing and preparation that may benefit the person after the death.

When all three perspectives are considered, the emerging picture seems to be a combination of depletion, relief, and anticipation effects (Schulz et al. 2008). For example, once the death occurs, the caregiver may feel extremely exhausted, but at the same time relieved that his or her loved one no longer has to suffer and that the immense strain of the caregiving role has ended and also may have had a chance to think about the impending death and their life afterward, possibly have a conversation with the loved one about these topics, and take care of some pragmatic necessities related to the death (e.g., financial planning, funeral arrangements).

The bulk of research studies to date indicate that many caregivers experience some sort of stress relief and/or benefit of anticipation rather than a depletion of their resources or at least that the first can outweigh the latter. However, bereavement outcomes following caregiving may also depend on how the loved one's end-of-life phase was experienced. For example, spouses of patients who died while on hospice care lived longer than spouses of patients who did not use hospice (Christakis and Iwashyna 2003). In contrast, family members were found to have poorer mental and physical health outcomes when aggressive treatments were performed at the patient's end of life (Wright et al. 2008). Besides consideration of such quality of life indicators characterizing the time preceding death, there is evidence that previous notions of anticipatory grief have been based on largely unfounded assumptions of the benefits of anticipatory grieving for post-death adaptation. Rather, findings have linked higher levels of pre-loss grief to higher levels of post-death grief (Liu and Lai 2006). This corresponds with the more general finding in the bereavement literature that those who experience high levels of distress before the death are also at risk of adjustment difficulties post-death (Bonanno et al. 2002; Schulz et al. 2006).

\section{Bereavement-Specific Theories}

Much of the theorizing discussed above involved conceptual models that could be applied to bereavement, but they were not developed specifically to account for people's reactions to the death of a loved one. For example, the stage model by Kübler-Ross was developed to describe the experience of dying persons, Bowlby's attachment model was originally designed to explain distress resulting from motherinfant separation, trauma theories addressed the case of various traumatic experiences, and the stress and coping approach dealt with any type of stressor. While coping with the death of a loved one has elements of all these areas, one could argue that there may be unique coping challenges that do not apply in the same way to other life experiences and that we therefore also need models specifically focusing on bereavement.

Two theoretical models reflecting this aspiration are Bonanno's four-component model (Bonanno and Kaltman 1999) and Stroebe and Schut's (1999, 2010) dual-process model. Bonanno's goal was to develop a conceptually sound and empirically testable framework for understanding individual differences in grieving. He identified four primary components of the grieving process - the context in which the loss occurs (e.g., was it sudden or expected, timely or untimely?), the subjective meanings associated with the 
loss (e.g., was the bereaved person resentful that he or she had to care for the loved one prior to the death?), changes in the representation of the lost loved one over time (e.g., does the bereaved person maintain a continuing connection with the deceased?), and the role of coping and emotion regulation processes that can mitigate or exacerbate the stress of loss. Bonanno's model makes the prediction that recovery is most likely when negative grief-related emotions are regulated or minimized and when positive emotions are instigated or enhanced (Bonanno 2001). This hypothesis, which is diametrically opposed to what would be derived from the psychodynamic approach, has generated considerable interest and support in recent years.

The dual-process model of coping with bereavement (DPM; Stroebe and Schut 1999, 2010) indicates that following a loved one's death, bereaved people alternate between two different kinds of coping: lossoriented coping and restoration-oriented coping. While engaged in loss-oriented coping, the bereaved person focuses on and attempts to process or resolve some aspect of the loss itself. Dealing with intrusive thoughts about the death is an example of loss-oriented coping. Restoration-oriented coping involves attempting to adapt to or master the challenges inherent in daily life, including life circumstances that may have changed as a result of the loss. Examples of restoration-oriented coping include distracting oneself from the grief, doing new things, or mastering new skills. Stroebe and Schut have proposed that bereaved individuals alternate between loss- and restoration-oriented coping and that such oscillation is necessary for adaptive coping. Hansson and Stroebe (2007) applied the DPM to the experience of bereavement in late life, cataloguing specific difficulties for older persons (such as the increased frequency and cumulative impact of multiple bereavements in the loss-oriented sphere and physical impairment, which may prevent the bereaved from carrying out the tasks that the deceased had taken care of, in the restorationoriented sphere).

\section{Outlook}

Today, the fundamental question facing bereavement theorists and researchers alike is the following: Why is it that some older adults are completely devastated by the death of a spouse, while others seem to emerge - sometimes after a period of intense suffering - relatively unscathed or even ultimately strengthened by what has happened? As noted above, accumulating evidence regarding variability in response to loss led researchers to move away from traditional grief models and instead employ frameworks, such as the stress and coping approach, Neimeyer's focus on meaning making, Bonanno and Kaltmann's four-component model, and the dual-process model, developed by Stroebe and Schut, each of which can account for divergent responses to loss. Drawing from these models, investigators are trying to identify risk and protective factors that influence the nature and course of grief following spousal loss. Below, we provide a brief summary of selected new directions that we think should be pursued to refine and expand conceptual thought in the field of bereavement.

To date, most of the bereavement literature has focused on adaptive processes within - rather than between - individuals. An intriguing question, however, is what happens to larger social units, such as families, when group members experience a shared loss in different ways (e.g., one family member expresses intense distress, whereas another shows less distress)? In such a case, would those who are more distressed be likely to benefit from the presence or availability of the less distressed person? Or would the lack of congruence in the experience of individual members lead to a mismatch and potential interference of coping efforts? Future work addressing these questions would make an important contribution because people rarely face a loss in a social vacuum (Stroebe et al. 2013). Theoretical models that do not address interpersonal processes in grieving lack this vital component of coping with loss. 
A related, also understudied, social context topic is the role of cultural influences on grief and bereavement. Ethnicity and cultural background have been found to be related to bereavement outcomes. For example, in a cross-cultural study, Chinese participants seemed to recover more quickly from bereavement emotionally compared to US Americans, but they also reported more somatic complaints (Bonanno et al. 2005). Culturally shaped spiritual convictions may also have an impact on how individuals deal with loss. For example, for a person who believes that life is suffering and death is transcendence, as seen in Zen Buddhism, the experience of loss may be easier to bear. There may also be culture-specific implications of particular losses. For example, in many cultures, losing one's husband involves loss of respect and basic human rights, which is likely to make coping more difficult, in particular for cohorts of older women who were not in a position to develop an autonomous lifestyle or status. Exploring in more detail whether and how the meanings associated with response to loss vary by culture might help us to gain a better understanding of the influence of culture-specific factors such as attitudes and expectations toward loss experiences.

Biological aspects of adaptation to a major loss could also be more important than expected so far (Curtis and Cicchetti 2003). There is some evidence for persistent alteration of stress mechanisms and brain functioning from early trauma. Early life stress apparently produces a sensitization of the cortical corticotropin-releasing neuronal system and the hypothalamic-pituitary-adrenal axis stress response, as well as structural and functional changes in the brain. Further, recent research has identified neurophysiological mechanisms linking stress to various negative consequences with respect to the immune, gastrointestinal, and cardiovascular systems (O'Connor 2013). Using physiological methods and the means of brain research could enrich ongoing efforts in psychosocial research on bereavement. Finally, combining physiological and psychosocial factors into more complex models to predict reactions to loss might allow us to better understand why some people are devastated by a loss like the death of a spouse, while others weather this kind of life event well or even experience positive development or personal growth in the context of major loss.

Theoretical advancement through more active consideration of areas such as interpersonal and physiological processes, as well as cultural influences, has the potential to deepen our understanding of grief and bereavement. This is particularly important for the case of complications in grieving. However, while recognizing symptoms of complications in grieving is critical to identifying individuals who are at risk for poor bereavement outcomes that persist over time so that adequate support sources can be allocated, the focus on symptom alone does help us understand why a person's response to loss may develop into a derailed adaptation process and perhaps even how this development could be prevented. Therefore, continued conceptual development in the field of bereavement is a vital effort.

\section{Cross-References}

- Care giving/Carer burden

- Depression

- Late Life Transitions

- Stress and Coping Theory in Geropsychology

- Theories to Understand Caregiver Stress and Coping

- Widowhood in Late Life 


\section{References}

Archer, J. (1999). The nature of grief: The evolution and psychology of reactions to loss. New York: Routledge.

Attig, T. (1996). How we grieve: Relearning the world. New York: Oxford University Press.

Baltes, M. M., \& Carstensen, L. L. (1996). The process of successful ageing. Ageing and Society, 16, 397-422. doi:10.1017/S0144686X00003603.

Boerner, K., \& Schulz, R. (2009). Caregiving, bereavement, and complicated grief. Bereavement Care, 28(3), 10-13. doi:10.1080/02682620903355382.

Bonanno, G. A. (2001). Grief and emotion: A social-functional perspective. In M. S. Stroebe \& R. O. Hansson (Eds.), Handbook of bereavement research: Consequences, coping, and care (pp. 493-515). Washington, DC: American Psychological Association.

Bonanno, G. A., \& Kaltman, S. (1999). Toward an integrative perspective on bereavement. Psychological Bulletin, 125, 760-786.

Bonanno, G. A., Wortman, C. B., Lehman, D., Tweed, R., Haring, M., Sonnega, J., ...Nesse, R. M. (2002). Resilience to loss, chronic grief, and their pre-bereavement predictors. Journal of Personality and Social Psychology, 83, 1150-1164.

Bonanno, G. A., Papa, A., Lalande, K., Zhang, N., \& Noll, J. G. (2005). Grief processing and deliberate grief avoidance: A prospective comparison of bereaved spouses and parents in the United States and the People's Republic of China. Journal of Consulting and Clinical Psychology, 73(1), 86-98. doi:10.1037/0022-0006X.73.1.86.

Bowlby, J. (1969). Attachment. (Attachment and loss, Vol. 1). New York: Basic Books.

Bowlby, J. (1973). Separation: Anxiety and anger. (Attachment and loss, Vol. 2). New York: Basic Books. Bowlby, J. (1980). Attachment and loss: vol 3: Loss: Sadness and depression. London: Hogarth Press.

Carr, D., Nesse, R., \& Wortman, C. (Eds.). (2006). Spousal bereavement in late life. New York: Springer.

Christakis, N. A., \& Iwashyna, T. J. (2003). The health impact of health care on families: A matched cohort study of hospice use by decedents and mortality outcomes in surviving, widowed spouses. Social Science \& Medicine, 57(3), 465-475.

Curtis, J. W., \& Cicchetti, D. (2003). Moving research on resilience into the 21st century: Theoretical and methodological considerations in examining the biological contributors to resilience. Development and Psychopathology, 3, 773-810. doi:10.1017/S0954579403000373.

Davis, C., Nolen-Hoeksema, S., \& Larson, J. (1998). Making sense of loss and benefiting from the experience: Two construals of meaning. Journal of Personality and Social Psychology, 75, 561-574.

Fraley, R. C., \& Shaver, P. R. (1999). Loss and bereavement: Bowlby's theory and recent controversies concerning "grief work" and the nature of detachment. In J. Cassidy \& P. R. Shaver (Eds.), Handbook of attachment theory and research: Theory, research, and clinical applications (pp. 735-759). New York: Guilford.

Freud, S. (1917/1957). Mourning and melancholia. In J. Strachey (Ed.), Standard edition of the complete work of Sigmund Freud. London: Hogarth Press.

Hansson, R. O., \& Stroebe, M. S. (2007). Bereavement in later life: Coping, adaptation, and developmental influences. Washington, DC: American Psychological Association Press.

Horowitz, M. (1986). Stress response syndromes. Northvale: Aronson.

Janoff-Bulman, R. (1992). Shattered assumptions: Towards a new psychology of trauma. New York: Free Press.

Jeter, K. (1983). Analytic essay: Family, stress and bereavement. In H. I. McCubbin, M. B. Sussman, \& J. M. Patterson (Eds.), Social stress and the family. New York: The Haworth Press.

Kübler-Ross, E. (1969). On death and dying. New York: Springer. 
Lazarus, R., \& Folkman, S. (1984). Stress, appraisal, and coping. New York: Springer.

Liu, N. C., \& Lai, E. Y. (2006). Find a way out: Bereavement support in Taiwan hospice. Support Care Cancer, 14, 4-10.

Maciejewski, P. K., Zhang, B., Block, S. D., \& Prigerson, H. G. (2007). An empirical examination of the stage theory of grief. JAMA: Journal of the American Medical Association, 297, 716-723.

Mikulincer, M., \& Shaver, P. (2013). Attachment insecurities and disordered patterns of grief. In M. Stroebe, H. Schut, \& J. van den Bout (Eds.), Complicated grief: Scientific foundations for health care professionals (pp. 190-203). London: Routledge.

Neimeyer, R. A. (1998). Lessons of loss: A guide to coping. Boston: McGraw-Hill.

Neimeyer, R. A. (2001). Meaning, reconstruction and the experience of loss. Washington, DC: American Psychological Association.

Neimeyer, R. A. (2006). Widowhood, grief and the quest for meaning: A narrative perspective on resilience. In D. Carr, R. M. Nesse, \& C. B. Wortman (Eds.), Spousal bereavement in late life (pp. 227-252). New York: Springer.

O'Connor, M.-F. (2013). Physiological mechanisms and the neurobiology of complicated grief. In M. Stroebe, H. Schut, \& J. van den Bout (Eds.), Complicated grief: Scientific foundations for health care professionals (pp. 204-218). London: Routledge.

Pearlman, L. A., Wortman, C. B., Feuer, C., Farber, C., \& Rando, T. (2014). Traumatic bereavement: A practitioner's guide. New York: Guilford Press.

Schulz, R., Boerner, K., Shear, K., Zhang, S., \& Gitlin, L. N. (2006). Predictors of complicated grief among dementia caregivers: A prospective study of bereavement. The American Journal of Geriatric Psychiatry, 14(8), 650-658.

Schulz, R., Boerner, K., \& Hebert, R. S. (2008). Caregiving and bereavement. In M. Stroebe, R. Hansson, H. Schut, \& W. Stroebe (Eds.), Handbook of bereavement research and practice: 21st century perspectives (pp. 265-285). Washington, DC: American Psychological Association Press.

Shaver, P. R., \& Tancredy, C. M. (2001). Emotion, attachment, and bereavement: A conceptual commentary. In M. S. Stroebe, R. O. Hansson, W. Stroebe, \& H. Schut (Eds.), Handbook of bereavement research: Consequences, coping, and care (pp. 63-88). Washington, DC: American Psychological Association.

Shear, K., Monk, T., Houck, P., Melhem, N., Frank, E., Reynolds, C., \& Sillowash, R. (2007). An attachment-based model of complicated grief including the role of avoidance. European Archives of Psychiatry and Clinical Neuroscience, 257, 453-461.

Stroebe, M. S. (1992-1993). Coping with bereavement: A review of the grief work hypothesis. Omega: Journal of Death and Dying, 26, 19-42.

Stroebe, M., \& Schut, H. (1999). The dual process model of coping with bereavement: Rationale and description. Death Studies, 23, 197-224.

Stroebe, M. S., \& Schut, H. (2001). Meaning making in the dual process model of coping with bereavement. In R. A. Neimeyer (Ed.), Meaning reconstruction and the experience of loss (pp. 55-73). Washington, DC: American Psychological Association.

Stroebe, M., \& Schut, H. (2010). The dual process model: A decade on. Omega: Journal of Death and Dying, 61, 273-291.

Stroebe, M. S., Hansson, R. O., Stroebe, W., \& Schut, H. (Eds.). (2001). Handbook of bereavement research: Consequences, coping, and care. Washington, DC: American Psychological Association.

Stroebe, M., Schut, H., \& Stroebe, W. (2005). Attachment in coping with bereavement: A theoretical integration. Review of General Psychology, 9, 48-66.

Stroebe, M., Folkman, S., Hansson, R. O., \& Schut, H. (2006). The prediction of bereavement outcome: Development of an integrative risk factor framework. Social Science and Medicine, 63, 2446-2451. 
Stroebe, M., Schut, H., \& Stroebe, W. (2007). Health consequences of bereavement: A review. The Lancet, 370, 1960-1973.

Stroebe, M., Schut, H., \& Boerner, K. (2013). Goals and over of the special issue bereavement: A family affair. Family Science, 4(1), 1-3. doi:10.1080/19424620.2013.819223.

Wortman, C. B., \& Silver, R. C. (1989). The myths of coping with loss. Journal of Consulting and Clinical Psychology, 57, 349-357.

Wright, A. A., Zhang, B., Ray, A., Mack, J. W., Trice, E., Balboni, T.,... Prigerson, H. G. (2008). Associations between end-of-life discussions, patient mental health, medical care near death, and caregiver bereavement adjustment. JAMA, 300(14), 1665-1673. doi:10.1001/jama.300.14.1665. 